\title{
Modelling vehicles kinematics and parking processes relevance on pollutant emissions in the city of Florence
}

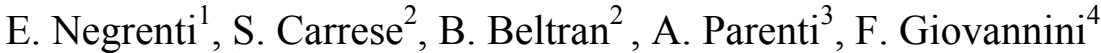 \\ \& V. Lapolla ${ }^{4}$ \\ ${ }^{1}$ ENEA Rome, Italy \\ ${ }^{2}$ University of Rome 3, Italy \\ ${ }^{3}$ ASTRAN s.r.l. Viterbo, Italy \\ ${ }^{4}$ ARPAT Florence, Italy
}

\begin{abstract}
The new version of TEE model (Transport Energy Environment) has been developed in the frame of the FP5 ISHTAR and HEARTS Projects with several features for better analysing transport related direct impacts, taking into account vehicles kinematics, cold start emissions distribution, parking processes, noise emissions and accident occurrence. Specific efforts were dedicated to the modelling of the effects of vehicles kinematics on hot emissions, where the software calculates link emissions by adopting average speed based functions, instantaneous emissions, or the innovative 'kinematics correction functions' model, and the modelling of parking processes which are relevant for cold start and evaporative emissions. In the frame of the testing and the application of the software version developed in the HEARTS Project, the tool was applied for the analysis of the effects of various transport measures in the city of Florence. The assessment of TEE software was performed in conjunction with a well-known dispersion model (OSPM) and the collection of air pollution measured data. The results obtained have shown the greater accuracy and sensitivities that can be achieved through the detailed modelling of key processes affecting vehicles emissions. Details of these results will be presented in the conference.
\end{abstract}

Keywords: transport policies assessment, vehicle emissions, urban planning, vehicle kinematics, parking. 


\section{Introduction}

Emissions from transport vehicles are modelled in a number of different ways, but this variability includes some 'fundamental rules' that give the 'essence' of emissions calculation: emissions are the sum of at least two main components: 'hot emissions' and 'cold start emissions'. If the modeller is interested in VOC emissions, then also the 'evaporative term' has to be added. Hot emissions are the emissions emitted when engine and abatement devices have reached a regime temperature. They are influenced by a number of parameters: vehicle kinematics, gradient of the road, altitude, maintenance level, vehicle age, vehicle loading, and electric loads. Normally models refer to hot emissions as a function of kinematics (e.g. average speed or instantaneous speed and acceleration) and then multiply the 'ideal' hot emission value for a number of corrective factors taking into account the other mentioned parameters (Negrenti [1]). Cold Start emissions are the emissions emitted from the start up until the vehicle reaches an almost steady state thermal condition. The cold start emissions affect in practice the first 3 or $4 \mathrm{kms}$ of trip and are particularly relevant for catalyst vehicles. Evaporative emissions are the emissions of unburned fuel from the 'weak points' of the vehicle: tank and canister. Current classic modelling recognises three different contributions to evaporative emissions: a) 'running emissions', emitted when vehicles are driven (emissions at tank level), b) 'hot soak' emissions, emitted from the canister at trip conclusion, and 'diurnal emissions', emitted at tank level by vehicles already parked. Evaporative emissions are a relevant fraction of total transport related VOC emissions, and so have a major role in the planning of measures for reducing VOC related pollution (e.g. critical benzene air pollution in Southern European cities). Two fundamental paths for increasing the accuracy of link based emission models are the more realistic modelling of vehicle kinematics and parking processes.

\section{Methodology}

The new version of TEE model (TEE-2005) allows to better analyse transport related direct impacts, taking into account vehicle kinematics, cold emission distribution, parking processes, noise emissions and accident occurrence (Negrenti [2]). As it regards vehicles kinematics, TEE calculates link emissions by adopting different options: average speed based emissions, instantaneous emissions, and the innovative 'kinematics correction functions' model describing both traffic flow condition and vehicle behaviour. The adoption of the kinematics correction has given very good results in the prediction of $\mathrm{CO}$ concentration levels in the city of Rome. For cold start emissions distribution, TEE now offers alternative solutions based on defaults of cold percentage depending on area type and day hour, or on the user input of either a link based cold fraction or the link related driven distance from trip origin. The parking process sub-model provides a meaningful treatment of traffic flows from and to parking areas and so allows one to better locate cold start vehicles emissions and evaporative emissions in space and time. The software now includes two fully 
new models: the noise emission model and the accident occurrence model. The former is sensible to vehicle speed and heavy duty vehicles presence. The accident model calculates the number of accidents involving only vehicles or vehicles and pedestrians and splits them by severity. These new models broaden the scope of TEE software from pure 'consumption and emissions' modelling to the area of the direct impacts of transport systems.

One of the most relevant developments for the accurate estimate of pollutant emissions distribution at urban level is the realisation of an 'advanced parking model'. Parking policy plays an important role (Hoglund [3]) in the traffic system and it affects the level of traffic congestion and environmental quality in most cities. This work focuses on a specific parking model for the accurate determination of emissions due to parking inlet and outlet processes as represented within the TEE modelling framework. The TEE model link representation is characterized by three 'flow modes' corresponding to transit, parking and inserting vehicles. The behaviour of the parking and inserting flows is described by means of simplified speed cycles, based on the kinematics of the vehicle during the parking and the insertion phases. The main parameters defining the shape of these cycles are searching speed, searching time and warm up time.

Parking search phase was represented in this work by dedicated 'searching speed' and 'searching time' models. The first model estimates searching speed (Young [4], Zadeh [5]) through a fuzzy approach as a function of the mean linear vehicle density and the occupation rate of the parking facility. The second model (Anderson [6], Van der Waerden et al [7]) uses a probabilistic approach to calculate the searching time based on the occupation rate of the link connected parking areas. The searching speed model and the searching time model are further divided into two sub-models depending on the category of parking: onstreet and off-street. As it regards the inserting phase, a large amount of pollutants is emitted due to the cold start effect. A set of models have been proposed in order to calculate the time employed by a car to be fully warmed up. Applications of the models showed that the parking model approach improves the sensibility of the TEE software. These specific models were integrated in the TEE 2005 Model in the framework of the EC FP5 HEARTS Project, and applied in the city of Florence by the ARPAT team.

\section{Model development and application results}

The two main developments recently obtained are here discussed: the kinematics modelling for transit flows and the modelling of parking.

One of the promising alternatives to the classically 'macroscopic' average speed emissions approach aims at obtaining a reasonably adequate kinematics description on the basis of easily available input data: the link average speed itself, a congestion indicator such as the 'lane flow density', link length and the fraction of green time at the intersection at the end of the link. The traffic density is used for calculating the fractions of time spent during cruising, acceleration, deceleration and idling phases. For low densities, traffic is smooth, 
very limited interactions occur among vehicles and all the time is spent in the cruise phase. For higher density values accelerations induced by interactions start, thus reducing time available for cruising. When density exceeds a threshold value, spontaneous breakdown occurs and short idling events take place. As density finally approaches the upper limit value we observe a fast decline of cruise time, a progressive reduction of time spent in accelerations and the rise of idling time until traffic is blocked at the density saturation value. The time spent during acceleration and deceleration is used to estimate the number of acceleration episodes on the basis of a driver behaviour hypothesis and acceleration rates derived from measured data. The minimum speed along the speed cycle can also be calculated from these data. The knowledge of the average speed and the time spent in cruising and in the other driving phases allows also the cycle cruising speed to be calculated. The overall result is a 'reconstructed' speed profile characterised by: a time interval spent in idling (only for high densities or presence of a traffic light), some accelerations and deceleration episodes between the minimum speed and the cruise speed, and a time interval spent at 'cruise' speed. This relatively simple solution describes the likely speed cycle far from the traffic light that is supposed (if any) at link end. The 'Kinematics Correction Function' (KCF) Model makes extensive use of the 'reconstructed cycle' algorithm. This approach assumes that the effect of speed variability can be expressed by means of a 'kinematics correction function'. The 'corrected' emission ' $E$ ' is obtained as the product of the 'average speed emission 'e' and the 'kinematics correction factor'. The KCF can be derived from a set of emission calculations in which both the 'reconstructed cycle' model and the average speed correlation are used. In order to derive a function of general applicability, the authors had to define a matrix of possible traffic conditions. By considering a discrete number of average speeds, of 'green time percent', of congestion levels represented by traffic density, and of different link lengths, it was possible to define a four-dimensional matrix of theoretical traffic situations for which the fuel consumption and the emissions of $\mathrm{CO}$ and VOC were calculated with both the approaches. This way the influence of the speed cycle can be quantified and the characterisation of the KCF as a function of speed, density, green time fraction and link length can be performed. The matrix used in the most recent recalculation of the KCF includes over 1300 different traffic conditions. The correlation between the KCF and the four input parameters was searched as the product of four independent functions:

$$
\mathrm{KCF}=\mathrm{K} 0 * \mathrm{~d}(\mathrm{D}) * \mathrm{~g}(\mathrm{G}) * 1(\mathrm{~L}) * \mathrm{v}(\mathrm{V})
$$

where $\mathrm{d}, \mathrm{g}, \mathrm{l}$, and $\mathrm{v}$ are classic mathematical functions of the variables $\mathrm{D}$ (traffic lane density, in vehicles $/ \mathrm{km}$ ), G (green time fraction), L (length, in $\mathrm{km}$ ) and $\mathrm{V}$ (Average Link Speed in $\mathrm{km} / \mathrm{hr}$ ), and $\mathrm{K} 0$ represents the value of the $\mathrm{KCF}$ coefficient for 'central' values of the four input variables.

TEE model adopting this correction function was assessed against CO measurements in Rome in the frame of the FP5 HEAVEN and ISHTAR Projects. 
Estimated CO levels were compared with the measured values in the Viale Libia monitoring station. Significant differences were found between the use of the $\mathrm{KCF}$ correction and the simple use of the COPERT III functions for hot emissions. The use of the correction largely provided the best results.

The daily profiles were predicted with very good approximation. The correlation of data calculated and measured was remarkably high (0.85). The calculated $\mathrm{CO}$ level without the adoption of the KCF correlation would have been sensibly lower (for the case of $\mathrm{CO}$ the $\mathrm{KCF}$ function ranges normally between 1 and 3).

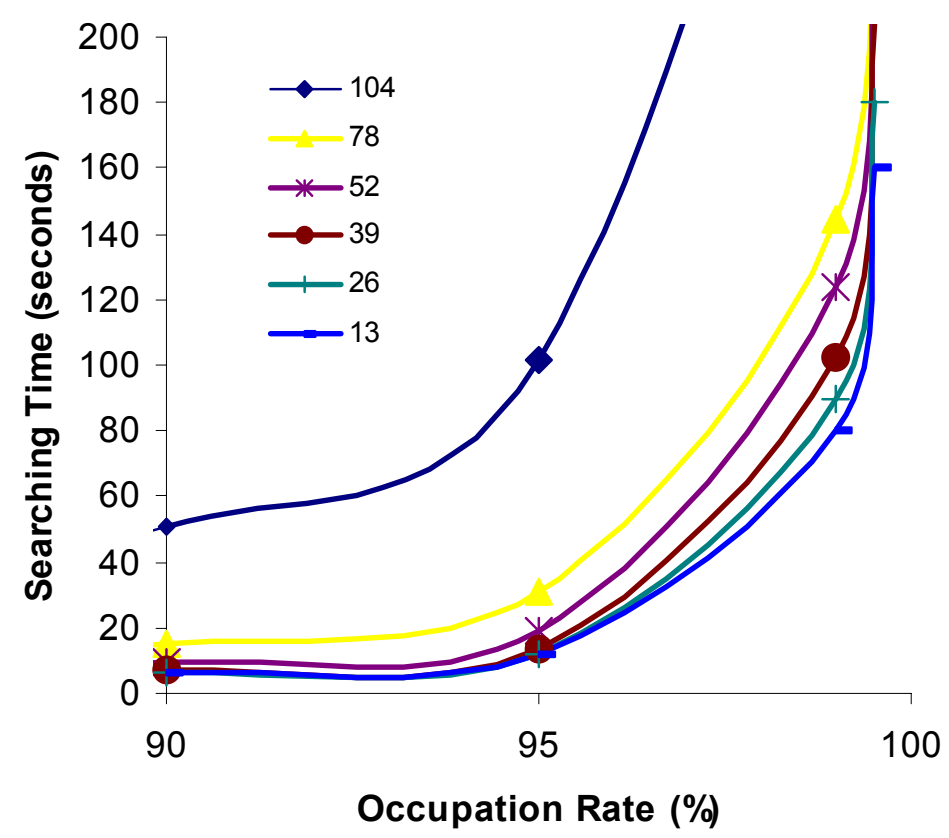

Figure 1: Searching time as a function of occupation rate for several densities.

The model of the searching time is then based on the analysis of probability number of attempts that a driver must carry out before finding a useful place to park. Relationship of searching time is the following:

$$
T_{\text {searching }}=\mu \frac{L_{p}}{V_{\text {searching }}}=\frac{L_{p}}{V_{\text {searching }}} \cdot \frac{N+1}{o c c_{2}-o c c_{1}} \cdot \log \left(\frac{N+1-o c c_{2}}{N+1-o c c_{1}}\right)
$$

where $\mu$ is the mean value of attempting, $\mathrm{N}$ is the total parking places, $\mathrm{L}_{\mathrm{p}}$ is the length of considered parking place, occ $_{1}$ is the number of occupied places in the beginning of the interval of study, occ 2 at the end of interval and $\mathrm{V}_{\text {searching }}$ is the speed of search. 
From parking model application a dependence emerges among the occupation rate, the searching time and the vehicle density. Searching time grows very slowly up to occupation rate of $95 \%$, then it increases quickly to a maximum value achieved when then parking is full.

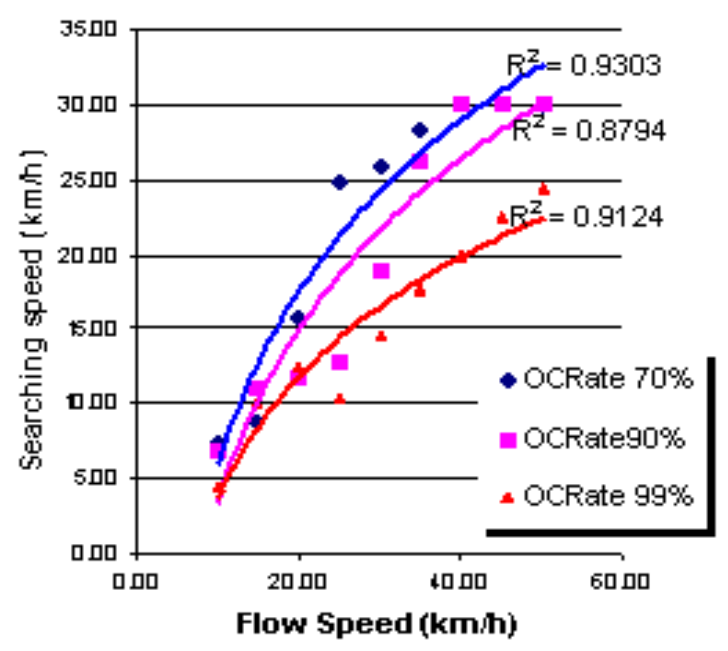

Figure 2: $\quad$ Search speed as a function of link speed and occupation rates.

To run TEE emission model it is necessary to complete the information obtained from traffic model EMME2 used in Florence. We need the traffic flow for the other hours of the day (hourly profiles) and the traffic flow for the other travel modes (i.e. two wheelers, duty vehicles, tourist coaches and extra-urban buses etc.). Moreover we need some data relating to the geometric characteristics of the links (e.g. link length, mean slope of link, etc.) and other data on traffic type of the link (link class, location and timing of traffic light, link area type, availability of parking and number of parking places etc.). In Table 1 we show the main information required for the application of TEE, with a short description of source of data used in the applications for the case study. Also, we denote the importance and the reliability of data (expert judgement). We point out that for some information the importance is different for the KCF (kinematics correction) or VM (average speed mode) options of TEE model. Each link has been classified according to traffic road categories. We used five road categories:

1) town entry/exit main roads

2) internal main roads

3) secondary roads in ZTL (controlled traffic zone, in the town central area)

4) secondary roads

5) highways and similar roads 
Table 1: $\quad$ Summary of the data used in TEE application.

\begin{tabular}{|c|c|c|c|}
\hline data & Source & reliability & importance \\
\hline flow & $\begin{array}{c}\text { EMME2 } \\
\text { output }+ \text { measurement }\end{array}$ & to test & high \\
\hline speed & $\begin{array}{c}\text { EMME2 } \\
\text { output }+ \text { measurement }\end{array}$ & to test & high \\
\hline lanes & EMME2 output & good & $\begin{array}{l}\text { high }(\mathrm{KCF}), \text { low } \\
\text { (otherwise) }\end{array}$ \\
\hline length & GIS calculation & good & high \\
\hline slope & GIS calculation & good & low \\
\hline fleet composition & $\begin{array}{c}\text { measurements (macro) }+ \\
\text { statistical data and local } \\
\text { corrections }\end{array}$ & good & high \\
\hline $\begin{array}{l}\text { cold start } \\
\text { percentage }\end{array}$ & $\begin{array}{l}\text { estimate from mobility } \\
\text { data }\end{array}$ & low & medium \\
\hline $\begin{array}{l}\text { parking and } \\
\text { inserting flow }\end{array}$ & $\begin{array}{c}\text { calculation from } \\
\text { EMME2 output }+ \\
\text { estimate }\end{array}$ & low & medium \\
\hline $\begin{array}{c}\text { properties of } \\
\text { distributed and } \\
\text { concentrated } \\
\text { parking areas } \\
\end{array}$ & $\begin{array}{c}\text { data from local } \\
\text { authorities + estimate }\end{array}$ & low & medium \\
\hline $\begin{array}{c}\text { traffic lights: cycle } \\
\text { and green } \\
\text { percentage }\end{array}$ & Default & low & $\begin{array}{c}\text { medium (KCF), } \\
\text { nothing (otherwise) }\end{array}$ \\
\hline
\end{tabular}

For each road category we know the hourly profiles of total vehicular flows; from direct measurements on sample roads we estimate the subdivision of total flows in sub-flows of macro-classes of vehicles. The number of vehicles belonging to micro-classes is obtained by splitting the total flow of a macro-class into single micro-classes flows according to the "weight" of the micro-class.

The mean speed at each hour is obtained from a flow-speed curve for each road category. In the lack of specific data from traffic model, the rate of cold start vehicles is estimated from mobility conjecture and data. For each link (and each hour of day) the cold fraction of vehicles is randomly drawn from a suitable range (with statistical uniform distribution). This range is obtained considering the length of daily mean trip, the total number of daily travels, the number of cold daily travels and the mean cold trip length. The rates of the inserting and parking vehicles on each link are estimated by processing output data of the traffic model. On each node of the network we calculated the balance of input and output vehicles from all the links connected to the node. The node is defined as "parking" or "inserting" whether input vehicles are larger than outgoing ones and vice versa. For parking nodes, the difference between input and output 
vehicles divided by the total number (input+output) of vehicles yields the percentage of parking vehicles assigned to the node considered. For inserting nodes we used the difference between output and input. It is clear that these values are a lower bound for link parking (inserting) vehicles. In addition to these data, a fixed rate for both inserting and parking vehicles is assigned to each link of the network. We have geo-referenced in a GIS framework the main concentrated parking areas of Florence town, with the related properties (number of car places, mean time of parking, hourly percentage of filling up etc.) obtained from data given from the local authority and management. Finally we have assigned link parking places by GIS calculation routine. For the policy scenario 'year 2010' we have used again the methods described above and many data were kept at the level of those of current scenario 'year 2003'. In synthesis, the most important changes in the forecast fleet are the insertion of future new micro-classes of vehicles according to EU standards and the very high reduction of the old 2-strokes mopeds (PRE EURO) with the increase of 4-strokes motorbikes; for the existing old micro-classes of vehicles at year 2003 (i.e. PRE EURO, EURO1, EURO2) we have assumed the reduction to be proportional to the number of vehicles in the micro-class, then we have used a yearly rate of reduction as the one between years 2002 and 2003. We have in general assumed to keep the same number of vehicles for a given macro-class.

Finally we tested the emission results of TEE KCF option and TEE "average speed" option. We compared the CO concentrations measured in an air quality monitoring station with the concentrations estimated by a dispersion model. We used the OSPM street canyon model (NERI, Denmark, OSPM 2000). The emissions provided by TEE KCF mode and TEE VM mode are used as input for the dispersion model. As an example, in Figure 3 and Figure 4, we show the hourly $\mathrm{CO}$ concentrations measured by the monitoring station and the OSPM results. The agreement between modelled and measured concentrations seems to be very good with $\mathrm{KCF}$ mode, but we have to take into account also the background concentration that is estimated to be about $0.5 \mathrm{mg} / \mathrm{m}^{3}$. For the same reason the difference between measured and 'average speed' option concentrations is not so big as shown. These results suggest that in the specific application of TEE emission model, the emissions from the real traffic on the street are inside a range between the two options of TEE model. In any case we recommend to run sensitivity studies on the most impacting uncertainties as those on the fraction of cold vehicles, the effects of vehicles aging, the poor knowledge of fleet composition for some categories. The consideration of the related variability can in facts significantly modify these conclusions.

\section{Conclusions}

New approaches have been developed for describing the effects of vehicle kinematics both in the transit flows and in the parking processes. These new models reduce the uncertainties in the description of the spatial and temporal distribution of emissions in all its main components : hot, cold start and evaporative. The application made in the city of Florence gave positive 
indications on the higher accuracy and sensitivity reached, but also suggested further areas of investigation and reduction of uncertainties for vehicles emissions modelling.

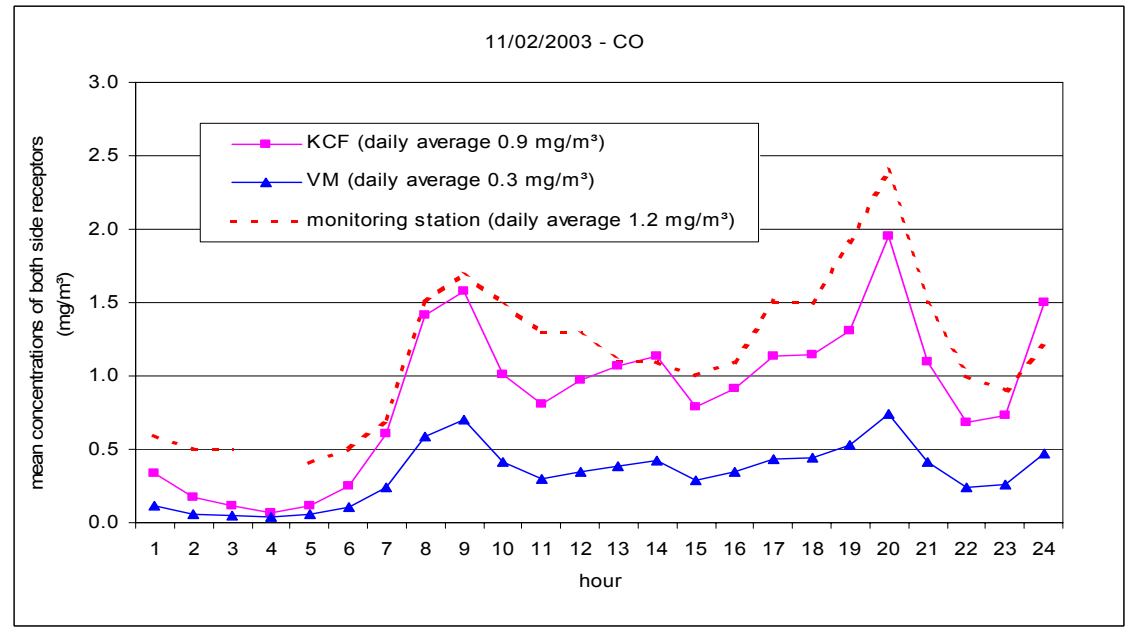

Figure 3: Hourly CO concentrations (February 2003) measured by an air monitoring station and those modelled by OSPM model and TEE with KCF (top) and average speed (bottom) kinematic options.

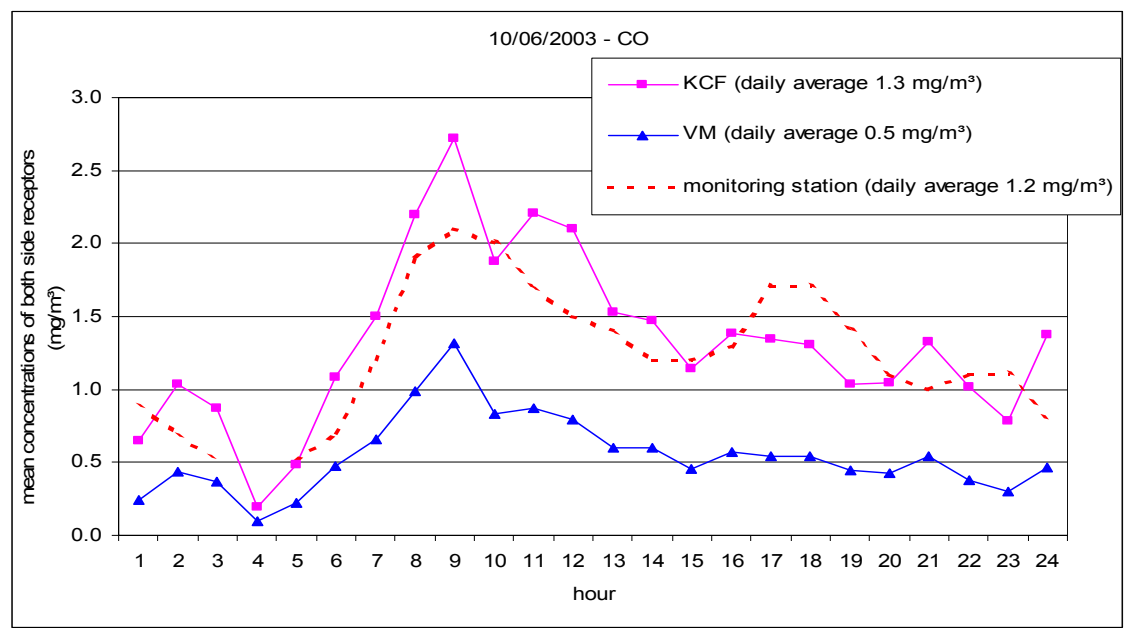

Figure 4: Hourly CO concentrations (June 2003) measured by an air monitoring station and those modelled by OSPM model and TEE with $\mathrm{KCF}$ (top) and average speed (bottom) kinematic options. 


\section{References}

[1] Negrenti, E. (1996) TEE: ENEA traffic emissions and energetic model micro-scale applications. The science of the total environment 189/190167-174.

[2] Negrenti, E. HEARTS Deliverable 7.3 - Issued by WHO - ECEH - Rome (2005).

[3] Hoglund, P. - Parking, energy consumption and air pollution. Science of the Total Environment 334-335 (2004) 39-45 - (2004).

[4] Young, W - The distribution of speed in parking facilities. Traffic Engineering \& Control. October 1988 - Vol. 29, No10. 504-513 - .(1988).

[5] Zadeh, L.A. - Fuzzy Sets a basis for a theory of probability. Fuzzy sets and systems. 1,3-28- (1978).

[6] Anderson S.P. - The Economics of on-street parking: Road Congestion and Driver Search. Journal of Urban Economics, 55(2004) 1-20 - (2004).

[7] Van der Waerden, $\mathrm{P}$ et al Travelers Micro-behaviors at Parking Lots: A model of Parking Choice Behaviors. TRB 2003 Annual Meeting CDROM- (2003).

[8] Negrenti, E et al. 'Modelling vehicles kinematics relevance in network based - urban emissions inventories' - Transport and Air Pollution Conference - Avignon - June 2003 - Le Collections de l'INRETS - Actes INRETS n. 92 - Vol.1 - P. 73 - ISSN 07690266 - ISBN 2-85782 5889 (2003). 